\title{
组织工程修复题下领关节的关键因素研究进展
}

王晨宇 ${ }^{1}$, 王英男 ${ }^{2}$, 汪存艺 ${ }^{1}$,施洁珺 ${ }^{2}$, 王慧明 ${ }^{2}$

1. 浙江大学医学院,浙江杭州 310058

2. 浙江大学医学院附属口腔医院浙江大学口腔医学院浙江省口腔生物医学重点 实验室, 浙江 杭州 310016

[ 摘 要] 颢下领关节骨关节炎(TMJOA)主要表现为颢下领关节盘(TMJD) 穿孔 以及髁突骨软骨复合体 (COCC) 破坏。近年来, 组织工程技术成为修复颢下领关节 的有效策略之一。随着支架材料技术的不断进步, 结合天然材料与人工合成材料 优势的复合支架成为优化支架性能的重要手段。近年来, 微创理念下的原位成胶 方法极大地解决了手术创伤以及材料吻合的问题, 有利于组织工程向临床转化。 细胞外基质支架技术在解决支架来源问题的同时最大程度地模拟了细胞外环境, 为题下领关节组织工程向动物水平的转化提供了重要的手段。由于肋软骨细胞来 源与扩增的限制, 采用不同来源的间充质千细胞成为题下领关节组织工程的广泛 选择, 其中从关节软骨表面分离得到的纤维软骨干细胞可能更为合适。转化生长 因子 $\beta$ 超家族由于其明确的骨软骨活性, 富血小板衍生物作为一种制备便捷的复合 生物因子, 同时结合间充质千细胞外泌体和应力刺激的形式调控细胞外微环境等 方法均在题下领关节组织工程中得到运用。未来, 通过复合生物活性因子并结合 一定的应力刺激可能成为颢下领关节组织工程研究的重要趋势之一。本文就组织 工程技术修复题下领关节骨软骨复合体及关节盘的进展, 尤其是在支架材料、种 子细胞以及刺激因子方面的研究进展作一综述, 以期为未来的研究设计和临床干 预提供指导。

[关键词 ] 颢下领关节; 骨关节炎; 组织工程; 题下领关节盘; 骨软骨复合体; 综述 [中图分类号］ Q819;R782.6［文献标志码］Ａ

\section{Research progress on tissue engineering in repairing temporo- mandibular joint}

WANG Chenyu, WANG Yingnan ${ }^{2}$, WANG Cunyi ${ }^{1}$, SHI Jiejun ${ }^{2}$, WANG Huiming ${ }^{2}$ (1. Zhejiang University School of Medicine, Hangzhou 310058, China; 2. Stomatology

\section{收稿日期: 2020-12-05 接受日期:2021-02-04}

基金项目: 浙江省基础公益研究计划(LGF21H140003); 浙江省医药卫生科技计划(2021418901); 中央高校基本科研业务 费专项资金(2020FZZX008-06)

第一作者:王晨宇,硕士研究生, 主要从事题下领关节组织工程研究;E-mail:21818694@zju.edu.cn; https://orcid.org/00000002-0671-0877

通信作者: 王慧明, 主任医师, 教授,博士生导师,主要从事口腔组织工程技术和口腔种植相关研究; E-mail : whmwhm@zju. edu.cn; https://orcid.org/0000-0002-1131-7455 
Hospital, School of Stomatology, Zhejiang University School of Medicine, Key Laboratory of Oral Biomedical Research of Zhejiang Province, Hangzhou 310006, China)

Corresponding author: WANG Huiming, E-mail: whmwhm@zju.edu.cn, https:/orcid.org/ 0000-0002-1131-7455

[ Abstract ] Temporomandibular joint osteoarthritis (TMJOA) is mainly manifested as perforation of temporomandibular joint disc(TMJD) and destruction of condylar osteochondral complex(COCC). In recent years, tissue engineering technology has become one of the effective strategies in repairing this damage. With the development of scaffold material technology, composite scaffolds have become an important means to optimize the performance of scaffolds with the combined advantages of natural materials and synthetic materials. The in situ gelling method with the minimally invasive concept can greatly solve the problems of surgical trauma and material anastomosis, which is beneficial to the clinical transformation of temporomandibular joint tissue engineering. Extracellular matrix scaffolds technology can solve the problem of scaffold source and maximize the simulation of the extracellular environment, which provides an important means for the transformation of temporomandibular joint tissue engineering to animal level. Due to the limitation of the source and amplification of costal chondrocytes, the use of mesenchymal stem cells from different sources has been widely used for temporomandibular joint tissue engineering. The fibrochondral stem cells isolated from surface layer of articular cartilage may provide one more suitable cell source. Transforming growth factor $\beta$ superfamily, due to its osteochondrogenesis activity has been widely used in tissue engineering, and platelet-rich derivative as a convenient preparation of compound biological factor, gradually get used in temporomandibular joint tissue engineering. With the deepening of research on extracellular microenvironment and mechanical stimulation, mesenchymal stem cells, exosomes and stress stimulation are increasingly being used to regulate the extracellular microenvironment. In the future, the combination of complex bioactive factors and certain stress stimulation may become a trend in the temporomandibular joint tissue engineering research. In this article, the progress on tissue engineering in repairing COCC and TMJD, especially in scaffold materials, seed cells and bioactive factors, are reviewed, so as to provide information for future research design and clinical intervention.

[ Key words ] Temporomandibular joint; Osteoarthritis; Tissue engineering; Temporomandibular joint disc; Osteochondral complex; Review

[J Zhejiang Univ (Med Sci), 2021, 50(2): 212-221.]

[ 缩略语] 髁突骨软骨复合体 (condylar osteochondral complex, COCC); 颡下领关节盘 (temporomandibular joint disc,TMJD); 颢下领关节骨关节炎(temporomandibular joint osteoarthritis, TMJOA); 聚乳酸一着基乙酸共聚物 [poly(lactic-co-glycolic acid), PLGA]; 转化生长因子(transforming growth factor, TGF); 间充质千细胞(mesenchymal stem cell, MSC); 骨髓间充质千细胞(bone marrow mesenchymal stem cell, BMSC); 骨形态发 生蛋白(bone morphogenetic protein,BMP); 富血小板血浆(platelet-rich plasma,PRP); 
富血小板纤维蛋白 (platelet-rich fibrin,PRF); 浓缩生长因子 (concentrate growth factors, CGF); 基质金属蛋白酶(matrix metalloproteinase,MMP); 牙髓干细胞(dental pulp stem cells, DPSC)

题下领关节是人体唯一能够双侧联动的关 节,其主要由下领骨髁突、颋骨关节窝以及其两者 之间的关节盘构成。如图 1所示, 下领骨髁突可分 为表面的关节软骨和软骨下骨两部分, 钙化软骨 层作为两者的交界面,具备骨与软骨的双重特征， 将骨与软骨紧密结合形成 COCC 来共同传递和分 散关节受到的生物力 ${ }^{[1]}$ 。TMJD 作为一种纤维软 骨组织, 其与关节软骨在胶原类型和机械性能上 有着明显的差别。关节软骨以 II 型胶原为主, 主 要承受压力负载, 而 TMJD 含有关节软骨内大部分 的 I 型胶原,需要承受压力与拉力负载。

TMJOA 的病理表现主要为关节软骨退行性 变和软骨细胞凋亡, 但其病损不仅局限于软骨, 更 包含了 TMJD 的变形和 COCC 的破坏。临床上 TMJOA 常引发关节的弹响及张闭口疼痛, 严重时 甚至出现前牙开合、下领后缩等症状。目前对 于 TMJOA 的治疗须结合患者的病情及病因分析, 可能采用包括保守治疗、关节腔内注射治疗、微创 关节镜手术以及开放性手术治疗等多种手段。研 究表明, 临床上 $80 \%$ 以上的患者采用局部热敷理 疗、药物治疗等保守方法, 在短期内可以明显缓解 症状 ${ }^{[2]}$, 但其长期疗效仍有待证明。关节腔内封 闭和抗炎药物注射以及关节镜下盘复位术疗效均 较好 ${ }^{[3-6]}$, 但其主要适用于盘髁形态未发生器质性 改变的 TMJOA 患者。对于已经出现关节盘穿孔、 髁突骨软骨破坏等组织缺损的患者, 临床上有必
要采用开放性的关节置换术来重建关节组织的形 态与功能。然而其手术难度较高, 术后还可能存 在骨关节炎复发等风险,不易被患者所接受。因 此,如何为题下领关节组织损伤的患者提供新的 治疗手段来修复关节盘和髁突骨软骨的破坏显得 尤为重要。

近年来, 随着组织工程理念的不断成熟, 利用 合适的支架材料荷载细胞或(和)因子成为了修复 骨关节炎领域的有效策略。为此,笔者综述了近 十年来题下领关节组织工程领域如何去重建具有 逐层过渡结构的 COCC 及具有生理功能的 TMJD, 尤其是在支架材料、种子细胞以及活性因子方面 的研究进展, 探讨组织工程在题下领关节组织修 复中的发展方向, 为未来的研究设计和临床干预 提供参考。

\section{1 组织工程支架材料}

理想的生物支架材料应具有一定的孔隙率并 最大限度地模拟细胞外环境, 为种子细胞提供可 支持其黏附和生长的荷载结构。在组织的修复过 程中, 支架不仅要具有良好的生物相容性, 其降解 速度也应与组织的再生速率相匹配。

目前,应用于颢下领关节组织工程的高分子 支架材料可以分为天然高分子材料、人工合成材 料、复合材料三大类。纤维蛋白 ${ }^{[7]}$ 、胶原蛋白 ${ }^{[8]}$ 、 透明质酸 ${ }^{[9]}$ 、琼脂糖 ${ }^{[10]}$ 等天然高分子材料大多能

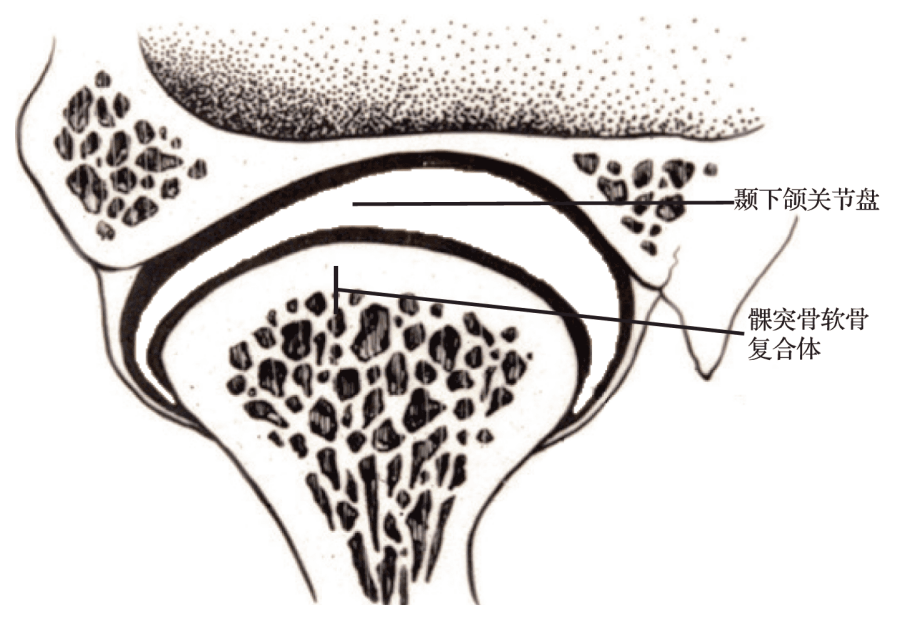

图 1 颞下领关节的解剖结构 (冠状切面) ${ }^{[1]}$

Figure 1 Anatomical structure of temporomandibular joint (frontal plane) ${ }^{[1]}$ 
够遇水膨胀, 形成三维网状交联聚合物。这些天 然水凝胶材料很好地模拟了细胞外基质环境, 与 细胞相互作用, 介导细胞的黏附。Wang 等 ${ }^{[8]}$ 将兔 TMJD 后外侧切除后植人重组胶原支架, 相较于未 植人组, 3 个月后可观察到再生纤维和细胞外基 质。然而, 由于天然材料降解速率不可控, 机械性 能不足, 越来越多的研究开始根据不同的需求人 工合成不同性能的支架材料。在关节盘组织工程 中最常用到的 $\mathrm{PLGA}^{[11-12]}$ 、聚己内酯 ${ }^{[13-14]}$ 支架可 以在体内完全降解成代谢产物, 其降解速率和机 械性能可以通过合成条件的调整进行控制。 Wang 等 ${ }^{[11}$ 通过静电纺丝技术合成了 PLGA 支架, 荷载题下领关节盘细胞与滑膜间充质干细胞膜 片, 在体外诱导后形成了 TMJD 类组织, 其软骨表 达和基质分泌明显升高。然而, 由于人工合成材 料对细胞的黏附与介导能力不足, 如何对其进行 表面的修饰和改良成为了支架材料的问题之一。 随着逐层纳米组装表面改性技术的发展, 利用二 氧化钛纳米薄膜修饰支架表面可增加细胞附着、 控制细胞分化表型。Ronald 等 ${ }^{[15]}$ 在体外研究中 发现, 增加二氧化钠纳米薄膜的厚度可使关节盘 软骨细胞的增殖水平和纤维性基质的合成能力 提高, 此外, 二氧化钛纳米薄膜的修饰可促进间 充质干细胞在材料表面的黏附和爬行 ${ }^{[16]}$ 。然而, 利用二氧化钛纳米薄膜修饰的支架效果有待动 物水平的研究。

人工合成材料和天然高分子材料形成的复合 支架, 可通过调整材料间的比例, 获得不同孔隙率 及组织降解速率的产物。Moura 等 ${ }^{[17]}$ 为了降低材 料的降解速率, 提高其机械性能, 合成了以聚乙二 醇丙烯酸酯水凝胶为核的聚己内酯支架, 以更好 地达到颢下领关节盘的强度。刘春栋等 ${ }^{[18]}$ 用透 明质酸改良聚乳酸支架, 在体外接种髁突软骨细 胞培养后发现透明质酸/聚乳酸组的软骨基质分 泌、软骨表达明显高于聚乳酸对照组。

近年来, 随着临床上微创理念的不断推广, 水 凝胶材料可通过注射方式在体内原位成胶, 成为 研究的热点之一。水凝胶材料本身具有较好的生 物安全性, 可作为细胞和因子的生物载体, 在体内 温度、酸碱度、离子等作用下形成一定形态的凝 胶 ${ }^{[19]}$ 。随着材料的降解, 负载的细胞和因子被不 断缓释, 已有动物研究观察到了膝关节软骨的修 复 ${ }^{[20-21]}$ 。这种方法解决了预塑性支架难于吻合的
缺陷以及开放性植人手术创伤过大的问题, 更易 向临床转化 ${ }^{[22]}$ 。在题下领关节组织工程领域, 郭 延伟等 ${ }^{[23]}$ 用可注射的透明质酸水凝胶负载成骨 多肽和硫酸软骨素, 这种支架材料荷载了 TGF$\beta 3$ 转染的脂肪干细胞,成功修复了兔髁突表面的 骨软骨缺损。张广德等 ${ }^{[24]}$ 利用 PLGA-聚乙二醇PLGA 三嵌段水凝胶负载肝细胞生长因子转染的 脂肪干细胞, 发现其对 TMJOA 修复作用明显。

在关节盘组织工程领域, 由于关节盘移植手 术的难度相对较高, 因此大多研究成果停留在体 外细胞水平, 有待进一步向动物体内转化。近年 来, 采用细胞外基质支架修复受损关节盘组织的 方法逐渐受到关注,这种完全取自损伤相应的生 物组织支架最大限度地保留了天然细胞外基质的 有效成分, 能提供比单纯天然材料或复合支架更 接近天然的细胞外微环境。去细胞处理也解决了 支架中的免疫原性问题, 拓宽了生物组织的获取 途径 ${ }^{[25]}$ 。Brown 等 ${ }^{[26]}$ 采用猪的脱细胞膀胱组织作 为支架, 在一侧关节盘切除的比格犬内成功形成 了类关节盘的功能性组织。Juran 等 ${ }^{[27]}$ 用激光处 理脱细胞的关节盘组织构建支架负载软骨分化的 脐带间充质干细胞, 在体外培养 3 周后观察到激 光处理的脱细胞关节盘支架更有利于细胞的黏附 和迁移,其机械性能更接近于天然关节盘组织。 尽管脱细胞组织支架能够最大限度地模拟组织结 构层次, 但在其支架处理过程中部分细胞外基质 和机械性能损失, 因此, 如何更好地优化组织脱细 胞处理方法, 将其与其他支架材料相结合来实现 TMJOA 的修复仍有待进一步研究。表1、2归纳整 理了文献中的支架材料 7 -15, 17-18,23-24,26-39]。

\section{2 组织工程种子细胞}

种子细胞作为组织工程的关键因素, 需要具 备良好的细胞特性和基质合成能力, 故其类型及 来源尤为重要。Vapniarsky 等 ${ }^{[29]}$ 在体外扩增异体 的肋软骨细胞, 通过聚合培养再分化的方法维持 扩增中软骨细胞性状的稳定, 在自组装琼脂糖中 构建组织工程移植物, 成功修复了穿孔的关节盘 组织, 改善了 TMJOA 评分。然而, 自体软骨细胞 的获取来源不足以及体外扩增过程中的性状不稳 定限制了其修复效果的提升 ${ }^{[40]}$ 。

MSC 作为一种生物体内分布广泛的多能干细 胞, 具备向成骨细胞、软骨细胞、脂肪细胞分化的 
表 1 关节盘组织工程支架材料研究一览

Table 1 Researches on tissue engineering scaffold materials of articular disc

\begin{tabular}{|c|c|c|c|c|c|}
\hline 作者(发表年) & 研究对象 & 材料种类 & 支架材料 & 细胞来源 & 生物因子 \\
\hline Kalpakci 等(2011 $)^{[10]}$ & 细胞 & 天然高分子材料 & 琼脂糖 & $\begin{array}{l}\text { 纤维软骨细胞、 } \\
\text { 关节软骨细胞 }\end{array}$ & 转化生长因子 $\beta 1$ \\
\hline MacBarb 等(2013) ${ }^{[28]}$ & 细胞 & 天然高分子材料 & 琼脂糖 & $\begin{array}{l}\text { 纤维软骨细胞、 } \\
\text { 关节软骨细胞 }\end{array}$ & $\begin{array}{c}\text { 软骨素酶 } \mathrm{ABC} \text { 、转化生 } \\
\text { 长因子 } \beta 1\end{array}$ \\
\hline Wang 等 $(2017)^{[8]}$ & 兔 & 天然高分子材料 & 重组胶原 & - & - \\
\hline Vapniarsky 等(2018) ${ }^{[29]}$ & 猪 & 天然高分子材料 & 琼脂糖 & 肋软骨细胞 & $\begin{array}{c}\text { 转化生长因子 } \beta 1 \text { 、软骨 } \\
\text { 素酶 } \mathrm{ABC} \text { 、赖氨酰氧 } \\
\text { 化酶样蛋白 } 2\end{array}$ \\
\hline Ahtiainen 等(2013) $)^{[30]}$ & 兔 & 人工合成材料 & 聚乳酸 & 脂肪来源干细胞 & 转化生长因子 $\beta 1$ \\
\hline Legemate 等(2016) & 细胞 & 人工合成材料 & 聚己内酯 & 骨髓间充质干细胞 & $\begin{array}{c}\text { 结缔组织生长因子、转 } \\
\text { 化生长因子 } \beta 1\end{array}$ \\
\hline Ronald 等(2016) ${ }^{[15]}$ & 细胞 & 人工合成材料 & 二氧化钛纳米薄膜 & 纤维软骨细胞 & - \\
\hline Wang 等 $(2018)^{[11]}$ & 细胞 & 人工合成材料 & $\begin{array}{l}\text { 聚乳酸-羟基乙酸共 } \\
\text { 聚物 }\end{array}$ & $\begin{array}{l}\text { 纤维软骨细胞、 } \\
\text { 滑膜间充质干细胞 }\end{array}$ & 转化生长因子 $\beta 3$ \\
\hline $\mathrm{Wu}$ 等 $(2014)^{[7]}$ & 裸鼠* & 复合材料 & 纤维蛋白一壳聚糖 & 滑膜间充质干细胞 & - \\
\hline Tarafder 等(2016) ${ }^{[14]}$ & 兔 & 复合材料 & $\begin{array}{l}\text { 聚乳酸-羟基乙酸微球- } \\
\text { 聚己内酯 }\end{array}$ & 骨髓间充质干细胞 & $\begin{array}{l}\text { 结缔组织生长因子、转 } \\
\text { 化生长因子 } \beta 3\end{array}$ \\
\hline Bousnaki 等 $(2018)^{[31]}$ & 细胞 & 复合材料 & 壳聚糖-海藻酸钠 & 牙髓干细胞 & - \\
\hline Moura 等(2020) ${ }^{[17]}$ & 材料 & 复合材料 & $\begin{array}{c}\text { 聚乙二醇丙烯酸酯水凝 } \\
\text { 胶为核的聚己内酯 }\end{array}$ & - & - \\
\hline Brown 等(2012) $)^{[26]}$ & 犬 & 组织支架材料 & 脱细胞膀胱组织 & - & - \\
\hline Juran 等(2015) $)^{[27]}$ & 细胞 & 组织支架材料 & 脱细胞颞下领关节盘 & 脐带间充质干细胞 & - \\
\hline
\end{tabular}

“一”:无相关资料; 体内异位再生.

潜力, 在组织工程领域受到了广泛的应用。在

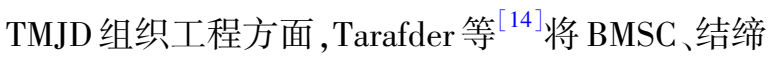
组织生长因子 / TGF- $\beta 3$ 的 PLGA 微球负载在聚己 内酯支架上, 促进了 TMJD 的愈合; 在 COCC 组织 工程领域, Zaki 等 ${ }^{[41]}$ 将 BMSC 注射至诱导的兔 TMJOA 关节腔内, 观察到 COCC 结构明显改善。

COCC 组织工程更加青棟于 BMSC 与关节软 骨细胞的利用, 而 TMJD 组织工程选用更加广泛的 种子细胞为来源于关节盘的纤维软骨细胞。尽管 不同组织来源的 MSC 都具有一定的多向分化能 力, 但在颢下领关节的修复中, 两者各有优势。其 中, BMSC 促进骨组织愈合的能力较强, 因此被广 泛应用于 COCC 组织工程中; 滑膜间充质干细胞 本身组织来源与题下领关节盘接近, 具有更强的 促进软骨向分化的能力; 脂肪间充质干细胞尽管 对于骨软骨缺损的修复效果不及其他 $\mathrm{MSC}^{[42]}$, 但 由于其获取途径更容易为临床所接受, 通过生物 因子的诱导, 对于题下领关节的修复也取得了一 定的成果 ${ }^{[23-24,31]}$ 。

随着对干细胞研究的不断深人, Dowthwaite
等 ${ }^{[43]}$ 在关节软骨表层分离并鉴定出一种能够自 我更新的软骨干细胞。Embree 等 ${ }^{[44]}$ 在题下领关 节发育过程中定位并分离了纤维软骨干细胞,其 不仅可以在体外向骨软骨向分化,还影响造血微 环境, 表明抑制 Wnt 信号通路可调控内源性纤维 软骨干细胞稳态进而修复骨软骨损伤, 但具体机 制仍有待进一步研究, 这一发现将为原位组织工 程修复骨软骨提供新的策略。沈佩 ${ }^{[33]}$ 通过外胚 层多阶段诱导促使胚胎干细胞向软骨干细胞分 化, 获得了与髁突软骨细胞较为接近的软骨干细 胞。负载软骨干细胞的凝胶海绵对于膝关节的骨 软骨缺损起到了明显修复作用,这一发现也将为 髁突骨软骨组织工程提供更为合适的种子细胞。

\section{3 组织工程刺激因子}

作为维持细胞外微环境不可或缺的一部分, 颢下领关节的修复更是离不开刺激因子,近年来 题下领关节组织工程应用广泛的活性因子主要有 TGF- $\beta$ 超家族 (TGF- $\beta 1 / 3$ 、BMP- 2 )、富血小板衍生 物(CGF、PRP、PRF) 等。 
表 2 髁突骨软骨复合体组织工程支架研究一览

Table 2 Researches on tissue engineering scaffold materials of condylar osteochondral complex

\begin{tabular}{|c|c|c|c|c|c|}
\hline 作者(发表年) & 研究对象 & 材料种类 & 支架材料 & 细胞来源 & 刺激因子 \\
\hline Zheng 等(2011) ${ }^{[32]}$ & 裸鼠* & 天然高分子材料 & 珊瑚 & 骨髓间充质干细胞 & $\begin{array}{l}\text { 碱性成纤维生长因子 } \\
\text { (转染) }\end{array}$ \\
\hline $\mathrm{Liu}$ 等 $(2011)^{[9]}$ & 兔 & 天然高分子材料 & 透明质酸水凝胶 & 无 & 胰岛素生长因子-1 \\
\hline 沈佩等 $(2019)^{[33]}$ & 大鼠 & 天然高分子材料 & 胶原蛋白 & 软骨干细胞 & - \\
\hline Dormer 等 $(2011)^{[12]}$ & 兔 & 人工合成材料 & $\begin{array}{l}\text { 聚乳酸-羟基乙酸共聚物微球 } \\
\text { 梯度 }\end{array}$ & 无 & $\begin{array}{l}\text { 骨形态发生蛋白-2、 } \\
\text { 转化生长因子 } \beta 1\end{array}$ \\
\hline 张广德等 $(2017)^{[24]}$ & 兔 & 人工合成材料 & $\begin{array}{l}\text { 聚乳酸-羟基乙酸共聚物-聚乙 } \\
\text { 二醇-聚乳酸-羟基乙酸共聚 } \\
\text { 物嵌段共聚物 }\end{array}$ & 脂肪间充质干细胞 & $\begin{array}{l}\text { 肝细胞生长因子 } \\
\text { (转染) }\end{array}$ \\
\hline Sun 等 $(2018)^{[34]}$ & 山羊 & 人工合成材料 & $\begin{array}{l}\text { 聚氧乙烯-聚氧丙烯醚嵌段共 } \\
\text { 聚物 }\end{array}$ & $\begin{array}{c}\text { 关节软骨细胞骨髓 } \\
\text { 间充质干细胞 }\end{array}$ & - \\
\hline 李祥等 $(2010)^{[35]}$ & 兔 & 复合材料 & 梯度磷酸三钲-胶原 & - & - \\
\hline 刘春栋等 $(2012)^{[18]}$ & 兔 ${ }^{*}$ & 复合材料 & 透明质酸-聚乳酸 & 关节软骨细胞 & - \\
\hline 石否等 $(2015)^{[36]}$ & 兔 & 复合材料 & 细胞膜片/PRF 双膜 & 骨髓间充质干细胞 & 富血小板纤维蛋白 \\
\hline Wei 等 $(2016)^{[37]}$ & 山羊 & 复合材料 & 差基磷灰石、脱细胞胁骨 & - & - \\
\hline 郭延伟等 $(2018)^{[23]}$ & 兔 & 复合材料 & $\begin{array}{l}\text { 成骨多肽-透明质酸-硫酸软 } \\
\text { 骨素 }\end{array}$ & 脂肪间充质干细胞 & $\begin{array}{l}\text { 转化生长因子 } \beta 3 \\
\text { (转染) }\end{array}$ \\
\hline Chin 等 $(2018)^{[38]}$ & 山羊 & 复合材料 & 聚癸二酸丙三醇酯/明胶 & - & 镁离子 \\
\hline Wang 等 $(2018)^{[39]}$ & 猪* & 复合材料 & $\begin{array}{l}\text { 骨相: 聚己内酯-羟基磷灰石 } \\
\text { 软骨相: 细胞膜片/聚乳酸-聚 } \\
\text { 羟基乙酸 }\end{array}$ & $\begin{array}{l}\text { 骨髓间充质干细胞 } \\
\text { 关节软骨细胞 }\end{array}$ & - \\
\hline
\end{tabular}

\section{“—”:无相关资料; “体内异位再生}

TGF- $\beta$ 超家族在骨软骨发育过程中发挥重要 作用, 是诱导 MSC 向骨软骨细胞方向分化的主要 活性因子 ${ }^{[45]}$ 。TGF- $\beta 1 / 3$ 可以在生理状态下通 过间变性淋巴瘤激酶 $5-\mathrm{Smad} 2 / 3$ 通路促进软骨细 胞的增殖, 上调 II 型胶原和蛋白聚糖的表 达 $^{[46]}$ 。Ying 等 ${ }^{[47]}$ 通过向 TMJOA 新西兰兔关节腔 内注射 TGF- $\beta 1$, 发现 TGF- $\beta 1$ 组的纤维软骨层结 构相对完整, 蛋白多糖的含量明显升高, 并没有出 现 TMJOA 相关的软骨退行性变与软骨下骨硬 化。Ahtiainen 等 ${ }^{[30]}$ 在体外用 TGF- $\beta 1$ 支架预处理 荷载自体脂肪干细胞的聚乳酸支架 1 周后植人到 题下领关节盘, 实验组未出现明显关节炎症及髁 突肥大。同样作为 TGF 家族中的一员, BMP-2/4 能够与细胞表面的受体作用激活 Smad 通路, 维持 软骨细胞的正常表型, 促进软骨细胞的生长与分 化 ${ }^{[48]}$ 。Dormer 等 ${ }^{[12]}$ 用 PLGA 微球荷载 BMP-2、 TGF- $\beta 1$,构建梯度渐变支架模仿骨至软骨的过渡, 修复了髅突骨软骨的缺损。

在软骨细胞分化成熟后, 持续 TGF- $\beta$ 作用会 激活下游 $\mathrm{Smad} 1 / 5 / 8$ 通路, 最终导致软骨细胞的 肥大和软骨内的钙化 ${ }^{[49]}$ 。 $\mathrm{Li}^{\text {等 }}{ }^{[50]}$ 在细胞水平证
明了 $\mathrm{Nel}$ 样分子 I 型可以改善 BMP、TGF- $\beta$ 在促软 骨分化中的不良反应, 并提高靶细胞对 B M P 、 TGF- $\beta$ 的敏感性。已有研究表明,关节腔内注射 $\mathrm{Nel}$ 样分子 I 型能够降低 TMJOA 的评分, 提高细 胞外基质的合成能力 ${ }^{[51]}$ 。因此, 软骨组织工程可 能需要协同多种活性因子的共同作用来改善单因 子可能带来的不良反应并提高其再生效率。

富血小板衍生物是将全血进行离心后得到的 富血小板的物质, 主要有 PRP、PRF、CGF 三类,富 血小板衍生物中含有血小板衍生生长因子、成纤 维细胞生长因子、TGF- $\beta$ 等多种生长因子, 可以促 进骨软骨组织的修复与再生。研究表明,关节腔 内注射 PRP 能够改善由物理、化学因素诱导的 TMJOA 症状 ${ }^{[52-53]}$ 。Nitecka-Buchta 等 ${ }^{[54]}$ 通过临床 试验证明 PRP 能够改善临床上 TMJOA 患者肌筋 膜疼痛的症状。

MSC 主要通过分化形成成骨细胞和成软骨细 胞实现骨软骨的修复,随着技术的发展与深人,发 现 MSC 可通过旁分泌调节骨软骨的微环境 ${ }^{[55]}$ 。 Zhang 等 ${ }^{[56]}$ 向 TMJOA 大鼠关节腔内注射 MSC 外 泌体, 改善了软骨细胞的基质表达和软骨下骨结 
构, 抑制了一氧化氮和 MMP-13 的产生, 实现关节 骨软骨的修复及再生。因此有研究将 BMSC 与题 下领关节盘细胞或软骨细胞共培养构建组织工程 材料 ${ }^{[11]}$, 然而其细胞间的信号介导机制仍有待进 一步研究。DPSC 是一类存在于牙髓组织中具有 较强的自我更新和多向分化潜能的间充质干细 胞。Bousnaki 等 ${ }^{[31]}$ 将 DPSC 搭载在壳聚糖/海藻 酸钠水凝胶中, 经过 8 周的体外培养后发现了明 显的纤维软骨组织再生, 其机械性能与天然关节 盘组织接近。Cui 等 ${ }^{[57]}$ 在研究中将 DPSC 注射至 TMJOA 大鼠的关节腔内, 观察到 DPSC 可以逆转 TMJOA 的炎性改变, 可能是通过抑制信号传导及 转录激活因子 1 通路下调 MMP- 3 和 MMP- 13 代谢 酶的表达。Ogasawara 等 ${ }^{[58]}$ 收集了 DPSC 的无血 清培养液用于 TMJOA 小鼠的静脉注射, 发现无血 清培养液组软骨细胞对于白介素- $1 \beta$ 、诱生型一氧 化氮合酶、MMP-13 的炎症表达明显降低, 软骨细 胞的调亡明显下调, 骨软骨组织得到修复与再生, 无血清培养液中含有大量生物活性因子。骆瑜 等 ${ }^{[59]}$ 分离并鉴定这些生物活性因子, 这种 DPSC 来 源的外泌体通过兔髁突表面骨软骨缺损模型也证 明了其对于软骨组织的修复能力。目前, 越来越 多的研究认识到了单一生物活性因子的局限性, 随着细胞间信号转导认识的不断深人, 通过复合 生物活性因子来更好地模拟细胞外微环境成为未 来颞下领关节组织工程研究的重要趋势之一。

卙下领关节在发挥其生理功能的过程中受到 周边组织带来的动态应力刺激 ${ }^{[60]}$, 过大的应力负 载会导致关节的退行性变, 而采用合适的强度和 持续时间进行机械刺激可促进关节软骨细胞分泌 细胞外基质, 提升新生软骨的机械性能。关节软 骨组织工程中采用的机械刺激主要包括直接压 力、静水压力、轴向张力、剪切力这四类 ${ }^{[61]}$ 。目前 研究较为广泛的主要是直接压力和静水压力, 可 能是因为轴向张力和剪切力往往与关节软骨的退 行性变相关。尽管有研究分别通过施加单轴张力 和剪切应力观察到了细胞外基质, 尤其是 II 型胶 原合成水平的提升,但其最佳的刺激强度和时间 仍有待进一步研究 ${ }^{[62-63]}$ 。

直接压力即直接负载在关节软骨表面的压 力, 也是关节软骨组织工程中最为常用的机械刺 激方式。不同研究团队对软骨细胞分别施加 $10 \mathrm{~g}$ 的静态直接压力和 $10 \% 1 \mathrm{~Hz}$ 的动态直接压
力, 均观察到机械性能及细胞外基质含量的提 高 ${ }^{[64-65]}$ 。天然的关节软骨在关节运动的过程中承 受 3 10 MPa 的静水压力, 主要来自于关节负荷时 滞留软骨基质内的蛋白多糖。另外, 静水压力本 身不会对组织造成剪切变形,避免机械刺激对细 胞外基质的破坏。因此,静水压力在关节组织工 程研究中得到了广泛的应用。李振强 ${ }^{[66]}$ 在体外 用琼脂糖构建自组装 TMJD 纤维软骨基体,并采用 静态静水压力和动态静水压力加压 1 周, 观察到 静水压力处理组的细胞外基质含量明显升高, 其 中动态静水压力组促进基质合成作用要优于静态 静水压力组。软骨缺乏血管而依赖于机械刺激进 行物质运输和信息传递, 不同类型和程度的机械 刺激效果及其与生物活性因子复合使用的意义有 待进一步探索。

\section{4 结 语}

随着分子生物学和材料学水平的不断进步, 题下领关节组织工程在支架材料、种子细胞以及 活性因子这三个方面发展迅速, 更为微创、便捷的 组织工程方法不断发现。未来的题下领关节组织 工程也应着眼于去更好地模拟生物体微环境, 包 括在复合支架的材料改性和应力复合生物因子诱 导方面,让关节盘和髁突骨软骨新生组织与天然 组织结构更为接近, 提高颢下领关节组织工程材 料的临床转化效能。

利益冲突 所有作者均声明不存在利益冲突

\section{参考文献}

[1] PETER E D. Functional occlusion: from TMJ to smile design $[\mathrm{M}]$. Philadelphia: Elsevier Mosby, 2007: 35.

[2] SIDEBOTTOM A J. Current thinking in temporomandibular joint management $[\mathrm{J}]$. Br J Oral Maxillofac Surg, 2009, 47(2): 91-94.

[3] 李春洁, 张一帆, 贾源源, 等. 透明质酸钠治疗颞下领 关节结构紊乱临床随机对照试验的系统评价 $[\mathrm{J}]$. 华 西口腔医学杂志, 2011, 29(5): 488-493.

LI Chunjie, ZHANG Yifan, JIA Yuanyuan, et al. Systematic review of the randomized controlled trial of sodium hyaluronate in the treatment of structural disorders of the temporomandibular joint $[\mathrm{J}]$. West China Journal of Stomatology, 2011, 29(5): 488493. (in Chinese)

[4] DYM H, BOWLER D, ZEIDAN J. Pharmacologic 
treatment for temporomandibular disorders $[\mathrm{J}]$. Dent Clin North Am, 2016, 60(2): 367-379.

[5] MACHADO E, BONOTTO D, CUNALI P A. Intraarticular injections with corticosteroids and sodium hyaluronate for treating temporomandibular joint disorders: a systematic review $[\mathrm{J}]$. Dental Press J Orthod, 2013, 18(5): 128-133.

[6] 张玲阁, 王章正, 张 点, 等. 关节镜手术治疗颞下领 关节紊乱病的疗效评价 $[\mathrm{J}]$. 医药论坛杂志, 2017, 38 (4): 96-97

ZHANG Lingge, WANG Zhangzheng, ZHANG Rui, et al. Efficacy evaluation of arthroscopic surgery for temporomandibular joint disorders $[\mathrm{J}]$. Journal of Medical Forum, 2017, 38(4): 96-97. (in Chinese)

[7] WU Y, GONG Z, LI J, et al. The pilot study of fibrin with temporomandibular joint derived synovial stem cells in repairing TMJ disc perforation $[\mathrm{J}]$. Biomed Res Int, 2014, 2014: 454021.

[8] WANG K H, CHAN W P, CHIU L H, et al. Histological and immunohistochemical analyses of repair of the disc in the rabbit temporomandibular joint using a collagen template $[\mathrm{J}]$. Materials, 2017, 10(8): 924.

[9] LIU X W, HU J, MAN C, et al. Insulin-like growth factor-1 suspended in hyaluronan improves cartilage and subchondral cancellous bone repair in osteoarthritis of temporomandibular joint $[\mathrm{J}]$. Int J Oral Maxillofac Surg, 2011, 40(2): 184-190.

[10] KALPAKCI K N, KIM E J, ATHANASIOU K A. Assessment of growth factor treatment on fibrochondrocyte and chondrocyte co-cultures for TMJ fibrocartilage engineering $[\mathrm{J}]$. Acta Biomater, 2011, 7(4): 1710-1718.

[11] WANG C H, WANG S, ZHANG B. et al. Layering poly (lactic-co-glycolic acid)-based electrospun membranes and co-culture cell sheets for engineering temporomandibular joint $\operatorname{disc}[\mathrm{J}]$. J Biol Regul Homeost Agents, 2018, 32(1): 55-61.

[12] DORMER N H, BUSAIDY K, BERKLAND C J, et al. Osteochondral interface regeneration of rabbit mandibular condyle with bioactive signal gradients $[\mathrm{J} / \mathrm{OL}]$. J Oral Maxillofac Surg, 2011, 69(6): e50-e57.

[13] LEGEMATE K, TARAFDER S, JUN Y, et al. Engineering human TMJ discs with protein-releasing 3D-printed scaffolds[J]. J Dent Res, 2016, 95(7): 800-807.

[14] TARAFDER S, KOCH A, JUN Y, et al. Micro-precise spatiotemporal delivery system embedded in 3D printing for complex tissue regeneration $[\mathrm{J}]$. Biofabrication, 2016, 8(2): 025003.

[15] RONALD S, MILLS D K. Fibrochondrocyte growth and functionality on $\mathrm{TiO}_{2}$ nanothin films $[\mathrm{J}]$. J Funct Biomater, 2016, 7(2): 15.

[16] KOMMIREDDY D S, SRIRAM S M, LVOV Y M, et al.
Stem cell attachment to layer-by-layer assembled TiO2 nanoparticle thin films $[\mathrm{J}]$. Biomaterials, 2006, 27 (24): 4296-4303.

[17] MOURA C, TRINDADE D, VIEIRA M, et al. Multimaterial implants for temporomandibular joint disc repair: tailored additive manufacturing production $[\mathrm{J}]$. Front Bioeng Biotechnol, 20208.

[18］刘春栋, 张志光, 苏 凯, 等. 透明质酸改性聚乳酸 支架组织工程软骨的构建 $[\mathrm{J}]$. 广东牙病防治, 2012, 20(3): 124-129.

LIU Chundong, ZHANG Zhiguang, SU Kai, et al. Construction of tissue engineering cartilage with modified polylactic acid scaffold using hyaluronic $\operatorname{acid}[\mathrm{J}]$. Journal of Dental Prevention and Treatment, 2012, 20(3): 124-129. (in Chinese)

[19] DIMATTEO R, DARLING N J, SEGURA T. In situ forming injectable hydrogels for drug delivery and wound repair $[$ J $]$. Adv Drug Deliver Rev, 2018, 127: 167-184.

[20] ZHOU T, LI X, LI G, et al. Injectable and thermosensitive TGF- $\beta 1$-loaded PCEC hydrogel system for in vivo cartilage repair[J]. Sci Rep, 2017, 7(1): 10553.

[21] MREDHA M T I, KITAMURA N, NONOYAMA T, et al. Anisotropic tough double network hydrogel from fish collagen and its spontaneous in vivo bonding to bone [J]. Biomaterials, 2017, 132: 85-95.

[22] RADHAKRISHNAN J, SUBRAMANIAN A, KRISH$\mathrm{NAN} \mathrm{U} \mathrm{M}$, et al. Injectable and 3D bioprinted polysaccharide hydrogels:from cartilage to osteochondral tissue engineering $[\mathrm{J}]$. Biomacromolecules, 2017, 18 (1): $1-26$.

[23] 郭延伟, 杨世茂. TGF- $\beta 3$ 转染脂肪干细胞复合 OGPHA-ChS 支架对兔髅突受损软骨的修复作用 $[\mathrm{J}]$. 上 海口腔医学, 2018, 27(6): 567-573.

GUO Yanwei, YANG Shimao. Effect of TGF- $\beta 3$ transfected with adipose derived stem cells and OGP-HA-chondroitin sulfate scaffold on repair of condylar cartilage in rabbits $[\mathrm{J}]$. Shanghai Journal of Stomatology, 2018, 27(6): 567-573. (in Chinese)

[24] 张广德, 李荣亮, 岳从雷, 等. 腺病毒介导 HGF 转染 脂肪干细胞复合温敏型可注射水凝胶对兔顷下领 关节骨关节病髁突软骨的修复作用 $[\mathrm{J}]$. 口腔医学研 究, 2017, 33(9): 924-927.

ZHANG Guangde, LI Rongliang, YUE Conglei, et al. Therapeutic effect of adenovirus-mediated HGF transfection of ADSCs combined with injectable hydrogei on the repair of temporomandibular joint arthritic condylar cartilage in rabbits $[\mathrm{J}]$. Journal of Oral Science Research, 2017, 33(9): 924-927. (in Chinese)

[25] YUAN X, WEI Y, VILLASANTE A, et al. Stem cell delivery in tissue-specific hydrogel enabled meniscal repair in an orthotopic rat $\operatorname{model}[\mathrm{J}]$. Biomaterials, 2017, 132: 59-71. 
[26] BROWN B N, CHUNG W L, ALMARZA A J, et al. Inductive, scaffold-based, regenerative medicine approach to reconstruction of the temporomandibular joint disk [J ]. J Oral Maxillofac Surg, 2012, 70(11): 2656-2668.

[27] JURAN C M, DOLWICK M F, MCFETRIDGE P S. Engineered microporosity: enhancing the early regenerative potential of decellularized temporomandibular joint dises[J]. Tissue Eng Part A, 2015, 21(3-4): 829-839.

[28] MACBARB R F, CHEN A L, HU J C, et al. Engineering functional anisotropy in fibrocartilage neotissues[J]. Biomaterials, 2013, 34(38): 99809989.

[29] VAPNIARSKY N, HUWE L W, ARZI B, et al. Tissue engineering toward temporomandibular joint disc regeneration $[J]$. Sci Transl Med, 2018, 10(446): eaaq1802.

[30 ] AHTIAINEN K, MAUNO J, ELLÄ V, et al. Autologous adipose stem cells and polylactide discs in the replacement of the rabbit temporomandibular joint $\operatorname{disc}[\mathrm{J}]$. J R Soc Interface, 2013, 10(85): 20130287.

[31] BOUSNAKI M, BAKOPOULOU A, PAPADOGIANNI D, et al. Fibro/chondrogenic differentiation of dental stem cells into chitosan/alginate scaffolds towards temporomandibular joint disc regeneration $[\mathbf{J}]$. J Mater Sci-Mater Med, 2018, 29(7): 97.

[32] ZHENG Y H, SU K, JIAN Y T, et al. Basic fibroblast growth factor enhances osteogenic and chondrogenic differentiation of human bone marrow mesenchymal stem cells in coral scaffold constructs $[\mathbf{J}]$. J Tissue Eng Regen Med, 2011, 5(7): 540-550.

[33] 沈 佩. 胚胎干细胞经外胚层向髁突软骨干细胞定 向分化及其软骨损伤修复应用研究 $[\mathrm{D}]$. 上海: 上海 交通大学, 2019.

SHEN Pei. Directional differentiation of embryonic stem cells into condylar cartilage stem cells via ectoderm and its application in cartilage injury repair [D]. Shanghai: Shanghai Jiao Tong University, 2019. (in Chinese)

[34] SUN H, HUANG Y, ZHANG L, et al. Co-culture of bone marrow stromal cells and chondrocytes in vivo for the repair of the goat condylar cartilage defects $[\mathrm{J}]$. Exp Ther Med, 2018, 16(4): 2969-2977.

[35] 李 祥, 查国庆, 朱双喜, 等. 胶原复合梯度磷酸三 钙修复髁突软骨缺损 $[\mathrm{J} / \mathrm{CD}]$. 中华口腔医学研究杂 志(电子版), 2010, 4(3): 239-243.

LI Xiang, ZHA Guoqin, ZHU Shuangxi, et al. Reconstruction of temporomandibular joint condylar cartilage defect with $\mathrm{Col} / \mathrm{TCP}[\mathrm{J} / \mathrm{CD}]$. Chinese Journal of Stomatological Research (Electronic Edition), 2010, 4 (3): 239-243. (in Chinese)

[36] 石 否, 李轶杰, 赵 萤, 等. NF- $\kappa \mathrm{B}$ 在压力调控
BMSCs/PRF 修复兔髁突软骨缺损中的作用研究 $[\mathrm{J}]$. 牙体牙髓牙周病学杂志, 2015, 25(3): 125-132, 178 . SHI Lei, LI Yijie, ZHAO Ying, et al. Role of NF-кB in condylar cartilage defects repair by pressure-regulated BMSCs/PRF in rabbits $[\mathrm{J}]$. Chinese Journal of Conservative Dentistry, 2015, 25(3): 125-132, 178. (in Chinese)

[37] WEI J, HERRLER T, HAN D, et al. Autologous temporomandibular joint reconstruction independent of exogenous additives: a proof-of-concept study for guided self-generation $[J]$. Sci Rep, 2016, 6(1): 37904.

[38] CHIN A R, GAO J, WANG Y, et al. Regenerative potential of various soft polymeric scaffolds in the temporomandibular joint condyle $[\mathrm{J}]$. J Oral Maxillofac Surg, 2018, 76(9): 2019-2026.

[39] WANG F, HU Y, HE D, et al. Scaffold-free cartilage cell sheet combined with bone-phase bmscs-scaffold regenerate osteochondral construct in mini-pig model [J]. Am J Transl Res, 2018, 10(10): 2997-3010.

[40 ] DUAN L, LIANG Y, MA B, et al. Epigenetic regulation in chondrocyte phenotype maintenance for cell-based cartilage repair[J]. Am J Transl Res, 2015, 7(11): 2127-2140.

[41] ZAKI A A, ZAGHLOUL M, HELAL M E, et al. Impact of autologous bone marrow-derived stem cells on degenerative changes of articulating surfaces associated with the arthritic temporomandibular joint: an experimental study in rabbits[J]. J Oral Maxillofac Surg, 2017, 75(12): 2529-2539.

[42] XIE X, WANG Y, ZHAO C, et al. Comparative evaluation of MSCs from bone marrow and adipose tissue seeded in PRP-derived scaffold for cartilage regeneration $[\mathrm{J}]$. Biomaterials, 2012, 33(29): 70087018.

[43] DOWTHWAITE G P, BISHOP J C, REDMAN S N, et al. The surface of articular cartilage contains a progenitor cell population[J]. J Cell Sci, 2004, 117 (6): 889-897.

[44] EMBREE M C, CHEN M, PYLAWKA S, et al. Exploiting endogenous fibrocartilage stem cells to regenerate cartilage and repair joint injury $[\mathrm{J}]$. Nat Commun, 2016, 7(1): 13073.

[45] CLEARY M A, VAN OSCH G J V M, BRAMA P A, et al. FGF, TGF $\beta$ and Wnt crosstalk: embryonic to in vitro cartilage development from mesenchymal stem cells $[J]$. J Tissue Eng Regen Med, 2015, 9(4): 332-342.

[46] VAN CAAM A, MADEJ W, GARCIA DE VINUESA A, et al. TGF $\beta 1$-induced SMAD2/3 and SMAD1/5 phosphorylation are both ALK5-kinase-dependent in primary chondrocytes and mediated by TAK1 kinase activity[J]. Arthritis Res Ther, 2017, 19(1): 112. 
[47] YING B, CHEN K, HU J, et al. Effect of different doses of transforming growth factor- $\beta 1$ on cartilage and subchondral bone in osteoarthritic temporomandibular joints $[$ J]. Bri J Oral Maxillofac Surg, 2013, 51(3): 241-246.

[48] YOON B S, POGUE R, OVCHINNIKOV D A, et al. BMPs regulate multiple aspects of growth-plate chondrogenesis through opposing actions on FGF pathways $[J]$. Development, 2006, 133(23): 46674678.

[49] HELLINGMAN C A, DAVIDSON E N B, KOEVOET $\mathrm{W}$, et al. Smad signaling determines chondrogenic differentiation of bone-marrow-derived mesenchymal stem cells: inhibition of Smad1/5/8P prevents terminal differentiation and calcification $[\mathrm{J}]$. Tissue Eng Part A, 2011, 17(7-8): 1157-1167.

[50] LI C S, ZHANG X, PÉAULT B, et al. Accelerated chondrogenic differentiation of human perivascular stem cells with NELL-1 $[J]$. Tissue Eng Part A, 2016, 22(3-4): 272-285.

[51] XIAO D, HU J, CHEN K, et al. Protection of articular cartilage by intra-articular injection of NEL-like molecule 1 in temporomandibular joint osteoarthritis [J/OL]. J Craniofac Surg, 2012, 23(1): e55-e58.

[52] KÜTÜK N, BA B, SOYLU E, et al. Effect of plateletrich plasma on fibrocartilage, cartilage, and bone repair in temporomandibular joint $[\mathrm{J}]$. J Oral Maxillofac Surg, 2014, 72(2): 277-284.

[53] COSKUN U, CANDIRLI C, KERIMOGLU G, et al. Effect of platelet-rich plasma on temporomandibular joint cartilage wound healing: experimental study in rabbits [J]. J Cranio-Maxillofac Surg, 2019, 47(2): 357-364.

[54] NITECKA-BUCHTA A, WALCZYNSKA-DRAGON K, KEMPA W M, et al. Platelet-rich plasma intramuscular injections - antinociceptive therapy in myofascial pain within masseter muscles in temporomandibular disorders patients: a pilot study $[\mathrm{J}]$. Front Neurol, 2019, 10: 250.

[55] MAUMUS M, MANFERDINI C, TOUPET K, et al. Adipose mesenchymal stem cells protect chondrocytes from degeneration associated with osteoarthritis $[\mathrm{J}]$. Stem Cell Res, 2013, 11(2): 834-844.

[56] ZHANG S, TEO K Y W, CHUAH S J, et al. MSC exosomes alleviate temporomandibular joint osteoarthritis by attenuating inflammation and restoring matrix homeostasis $[\mathrm{J}]$. Biomaterials, 2019, 200: 35-47.

[57] CUI S J, ZHANG T, FU Y, et al. DPSCs attenuate experimental progressive TMJ arthritis by inhibiting the STAT1 pathway[J]. J Dent Res, 2020, 99(4): 446455.

[58] OGASAWARA N, KANO F, HASHIMOTO N, et al. Factors secreted from dental pulp stem cells show multifaceted benefits for treating experimental temporomandibular joint osteoarthritis $[\mathrm{J}]$. Osteoarthritis Cartilage, 2020, 28(6): 831-841.

[59］骆 瑜, 李若涵, 彭友俭. 牙髓干细胞来源外泌体修 复兔颢下领关节软骨损伤后软骨组织修复的实验 研究 $[J]$. 临床口腔医学杂志, 2020, 36(4): 202-205. LUO Yu, LI Ruohan, PENG Youjian. Cartilage tissue regeneration after dental cartilage injury induced by exosome derived from dental pulp stem cells in rabbits [J]. Journal of Clinical Stomatology, 2020, 36(4): 202-205. (in Chinese)

[60 ] LI Q, REN S, GE C, et al. Effect of jaw opening on the stress pattern in a normal human articular disc: finite element analysis based on MRI images $[\mathrm{J}]$. Head Face Med, 2014, 10(1): 24.

[61 ] SALINAS E Y, HU J C, ATHANASIOU K. A guide for using mechanical stimulation to enhance tissueengineered articular cartilage properties $[\mathrm{J}]$. Tissue Eng Part B-Rev, 2018, 24(5): 345-358.

[62 ] LEE J K, HUWE L W, PASCHOS N, et al. Tension stimulation drives tissue formation in scaffold-free systems [J]. Nat Mater, 2017, 16(8): 864-873.

[63] PEI Y, FAN J J, ZHANG X Q, et al. Repairing the osteochondral defect in goat with the tissue-engineered osteochondral graft preconstructed in a double-chamber stirring bioreactor $[J]$. Biomed Res Int, 2014, 2014: 219203.

[64] MACBARB R F, PASCHOS N K, ABEUG R, et al. Passive strain-induced matrix synthesis and organization in shape-specific, cartilaginous neotissues $[\mathrm{J}]$. Tissue Eng Part A, 2014, 20(23-24): 3290-3302.

[65] MAUCK R L, SOLTZ M A, WANG C C, et al. Functional tissue engineering of articular cartilage through dynamic loading of chondrocyte-seeded agarose gels[J]. J BioMech Eng, 2000, 122(3): 252-260.

[66］李振强. 自组装额下领关节盘纤维软骨模型的构 建及静水压对其基质合成的影响 [D].兰州: 兰州 大学,2011.

LI Zhenqiang. Construction of self-assembled disc fibrocartilage model of temporomandibular joint and the effect of hydrostatic pressure on its matrix synthesis[D]. Lanzhou: Lanzhou University, 2011. (in Chinese)

[本文编辑 沈 敏 余 方] 